\title{
Coupled Noisy Spiking Neurons as Velocity-Controlled Oscillators in a Model of Grid Cell Spatial Firing
}

\author{
Eric A. Zilli and Michael E. Hasselmo \\ Center for Memory and Brain, Department of Psychology, and Program in Neuroscience, Boston University, Boston, Massachusetts 02215
}

One of the two primary classes of models of grid cell spatial firing uses interference between oscillators at dynamically modulated frequencies. Generally, these models are presented in terms of idealized oscillators (modeled as sinusoids), which differ from biological oscillators in multiple important ways. Here we show that two more realistic, noisy neural models (Izhikevich's simple model and a biophysical model of an entorhinal cortex stellate cell) can be successfully used as oscillators in a model of this type. When additive noise is included in the models such that uncoupled or sparsely coupled cells show realistic interspike interval variance, both synaptic and gap-junction coupling can synchronize networks of cells to produce comparatively less variable network-level oscillations. We show that the frequency of these oscillatory networks can be controlled sufficiently well to produce stable grid cell spatial firing on the order of at least 2-5 min, despite the high noise level. Our results suggest that the basic principles of oscillatory interference models work with more realistic models of noisy neurons. Nevertheless, a number of simplifications were still made and future work should examine increasingly realistic models.

\section{Introduction}

Following the initial discovery and characterization of grid cells in rat entorhinal cortex (Fyhn et al., 2004; Hafting et al., 2005; Sargolini et al., 2006), a number of models of the spatial firing properties of these cells were offered. Generally, the mechanisms of the models fall into two categories: continuous attractors (Fuhs and Touretzky, 2006; McNaughton et al., 2006; Guanella et al., 2007; Burak and Fiete, 2009) and oscillatory interference (O'Keefe and Burgess, 2005; Blair et al., 2007, 2008; Burgess et al., 2007, 2008; Hasselmo et al., 2007; Hasselmo, 2008) (but see Gaussier et al., 2007; Kropff and Treves, 2008).

Oscillatory interference models were first used to explain hippocampal place cell phase precession (O'Keefe and Recce, 1993; Lengyel et al., 2003; Huhn et al., 2005) by combining two oscillators to produce an interference pattern of activity in the spiking of a neuron. Grid cell models simply add one or more additional oscillators to produce a two-dimensional (2D) interference pattern. One oscillator maintains an arbitrary baseline frequency and the remaining, active oscillators are driven to various frequencies above or below the baseline frequency. The specific frequencies used at each time are based on the animal's velocity in different directions through a simple transformation that results in the phase difference between an active oscillator and the baseline oscillator encoding one-dimensional (1D) positional information. Active oscillators maintaining 1D positional information along di-

Received Jan. 26, 2010; revised Aug. 20, 2010; accepted Aug. 26, 2010.

This work was supported by National Institute of Mental Health Silvio 0. Conte Center Grant MH71702, and R01 Grants MH61492 and MH60013; National Science Foundation Science of Learning Center Grant SBE 0354378 (CELEST); and the Office of Naval Research. We thank Lisa Giocomo and Jim Heys for helpful comments.

Correspondence should be addressed to Eric A. Zilli, Center for Memory and Brain, 2 Cummington Street, Boston University, Boston, MA 02215. E-mail: zilli@bu.edu.

DOI:10.1523/JNEUROSCI.0547-10.2010

Copyright $\odot 2010$ the authors $\quad 0270-6474 / 10 / 3013850-11 \$ 15.00 / 0$ rections $60^{\circ}$ apart produce a regular hexagonal interference pattern in space.

One prominent criticism of these models (Giocomo and Hasselmo, 2008; Hasselmo, 2008; Welinder et al., 2008; Burak and Fiete, 2009; Zilli et al., 2009) is that they are presented in terms of abstract, perfect oscillators, whereas oscillators in the brain are noisy and have more complicated dynamics. Zilli et al. (2009) showed that experimentally measured variability in the examined biological oscillators was large enough that an oscillatory interference model's spatial firing would be expected to remain stable for a few seconds at best. This strongly supported the noise criticism, but there are at least two ways for these models to overcome this problem. One possibility is that sensory cues can frequently or even constantly reset the grid network (Redish and Touretzky, 1997; Redish, 1999; Burgess et al., 2007; Samu et al., 2009). A second possibility, as suggested by Zilli et al. (2009), is that these individually noisy oscillators may be coupled in vivo, and through synchronization the network's oscillations may be less variable (Manor et al., 1997; Needleman et al., 2001; Ly and Ermentrout, 2010; Tabareau et al., 2010).

To test the latter solution, we use numerical simulations of two different neural models and verify that coupled, noisy neural oscillators can be used successfully in the oscillatory interference framework. We close with discussion of our assumptions as well as implications and directions for future study.

\section{Materials and Methods}

Computational methods. Simulations were performed in MATLAB 7.0.0.19920 (R14). MATLAB source code to reproduce all figures and to support many claims labeled preliminary simulations or unpublished observations is available on ModelDB. Equations of the models were solved using the forward Euler method with a time step of $d t=0.1 \mathrm{~ms}$ (simple model) or $d t=0.01 \mathrm{~ms}$ (biophysical model). Of these two models, only one was used to model the velocity-controlled oscillators 
A

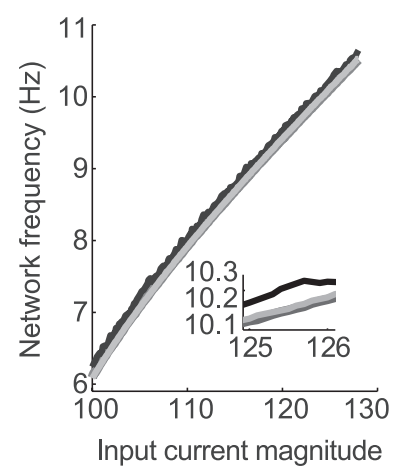

B

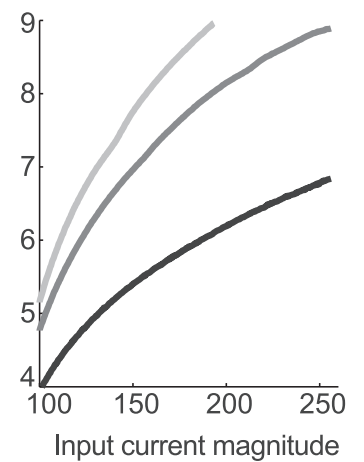

C

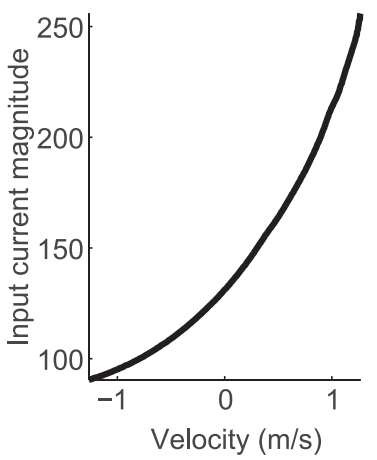

Figure 1. Firing frequency versus input current for individual and networks of simple model cells. $A$, The firing frequency of an individual cell without noise (light gray) is very similar to the response of an individual noisy cell (black), but even more similar to a network of 250 noisy, gap-junction-coupled neurons (dark gray). For clarity, an inset with an expanded $y$-axis is shown. $\boldsymbol{B}, A$ network of 250 noisy, synaptically coupled neurons fires at much lower frequencies than any of the cells in $\boldsymbol{A}$ for the same input magnitude. The entire $F(I)$ curve also moves downward as the synaptic coupling strength $g$ is increased ( $g=192$, light gray; $g=256$, dark gray; $g=384$, black) (compare with supplemental Fig. S4A, available at www.jneurosci.org as supplemental material). C, Since the firing rate, $f$, is directly related to velocity, $v, v=\left(f-\omega_{\mathrm{b}}\right) / \beta$, the inverse of the $F(I)$ curve (shown for the synaptically coupled network) describes the input an oscillator network requires to encode different velocities along its preferred direction. General parameters: $15 \mathrm{~s}$ simulations and see Table 1 . Single unit: $n=1, w=1.2, \sigma=0$, or $\sigma=100$. Gap-junction coupling: $n=250, w=0.0048, \sigma=100$. Synaptic coupling: $n=$ $250, w=0.0048, \sigma=100, \omega_{\mathrm{b}}=6.4405 \mathrm{~Hz}$.

(VCOs) in any particular simulation. Preliminary simulations showed these step sizes were sufficiently small. As our simulations often comprised hundreds of cells $(15,000$ in the largest simulation) and were up to $320 \mathrm{~s}$ long, it was important for processing time that the step size not be too small. The grid cell itself was modeled as a leaky integrateand-fire (LIF), resonate-and-fire, or simple model neuron, and it was simulated alongside either the simple model or the biophysical model VCOs, using the same size time step the oscillator model used.

Spike times were determined by comparing the voltage variable with a fixed value (thresholds of 1 for the LIF model, 1 for the resonate-and-fire model, $\theta_{\text {spike }}$ for the biophysical model, and a peak value $v_{\text {peak }}$ for the simple model). For all models, a spike was recorded on the time step where the voltage crossed the threshold from below (and for the LIF, resonate-and-fire, and simple models, the voltage was then immediately set to the reset voltage, as specified below).

To provide two-dimensional trajectories as input for our simulations, we used experimentally collected rat trajectory data from Hafting et al. (2005) (available for download at http://www.ntnu.no/cbm/moser/ gridcell). The trajectory data are a set of coordinates, $x_{\exp }(t)$ and $y_{\exp }(t)$, sampled at resolution $d t_{\exp }=0.02 \mathrm{~s}$ (note that some trajectory files seem to contain multiple, concatenated trajectories separated by a discontinuity). The difference between adjacent position samples was used as the velocity input to the simulation. Simulations were performed at a finer temporal resolution than $d t_{\text {exp }}$, so the velocity signals were linearly interpolated, producing velocity signals $v_{\mathrm{x}}(t)$ and $v_{\mathrm{y}}(t)$.

To adjust the smoothness of the trajectory, $v_{\mathrm{x}}(t)$ and $v_{\mathrm{y}}(t)$ were bidirectionally (in time) low-pass filtered with a third-order Butterworth filter at $0.4 \mathrm{~Hz}$ (an arbitrary value controlling the smoothness of the trajectory). Most of our 2D simulations used the filtered velocity input, as the unfiltered velocity results were often poorer in comparison. Which of the two cases is more realistic is unclear, as the experimental trajectory recording likely contains high-frequency jitter from the video tracking system and the vestibular and proprioceptive systems of the animal themselves may also act to low-pass filter their acceleration or velocity signals to prevent small movements of the head from being path integrated. From the trajectory, we can calculate two spatial functions that are of frequent interest: speed, $s(t)=\sqrt{\left[v_{\mathrm{x}}(t) d t\right]^{2}+\left[v_{\mathrm{y}}(t) d t\right]^{2}}$; and direction $\phi(t)=\operatorname{atan}\left[v_{y}(t) / v_{x}(t)\right]$, where atan is the four quadrant arctangent. Filtering decreases the velocity of the trajectory. For example, in $320 \mathrm{~s}$ of trajectory prepared in this manner, the mean (peak) instanta-

neous speed is $22 \mathrm{~cm} / \mathrm{s}(89 \mathrm{~cm} / \mathrm{s})$ in the unfiltered trajectory compared with $17 \mathrm{~cm} / \mathrm{s}$ (49 $\mathrm{cm} / \mathrm{s}$ ) in the filtered trajectory.

Our simulations required knowing the frequency response, $F(I)$, of the oscillator cell or network to current injections. In most cases, $F(I)$ was calculated numerically using input magnitudes at fixed intervals to produce a resolution of $\sim 0.02 \mathrm{~Hz}$ over ranges of at least $4 \mathrm{~Hz}$ and other values were linearly interpolated. For the $n=5000$ case, a resolution of $\sim 0.15 \mathrm{~Hz}$ was used. A range of $4 \mathrm{~Hz}$ was needed because we used $\beta=2 \mathrm{~Hz} /(\mathrm{m} / \mathrm{s})$ (see below) and allowed for a maximum instantaneous velocity of 1 $\mathrm{m} / \mathrm{s}$, thus requiring $2 \mathrm{~Hz}$ above and below the baseline frequency. When the networks comprised noisy neurons, the measured $F(I)$ curve became noisy, which imposed a maximum level of accuracy (Fig. 1A, black line, uncoupled noisy cell).

We also simulated abstract VCOs $\Phi_{\mathrm{i}}$ and grid cells using a common form of the oscillatory interference model (Eq. 1). The abstract model was simulated using the forward Euler method using the same time resolution as our network model (which depended on the neural model in use). Each abstract VCO's state was characterized by its phase $\varphi_{\mathrm{i}}$ evolving at a timevarying frequency, $f_{\mathrm{i}}(t)=\omega_{\mathrm{b}}+\beta s(t) \cos \left[\phi_{i}-\right.$ $\phi(t)]$, which was a function of speed, $s(t)$; body direction $\phi(t)$; and each VCO's preferred direction $\phi_{\mathrm{i}}$.

A quantitative measure of the phase of the network oscillators was desired, but translating the state of a neuron into the corresponding phase is not a trivial task and translating the state of a network of coupled neurons into a population phase is harder still. Generally, each point in the neuron's state space can be identified with an asymptotic phase (Izhikevich, 2007), but finding the phase of an arbitrary point is computationally intensive. Instead of identifying phases in this manner, we simulated an abstract VCO $\Phi_{\mathrm{i}}$ alongside each network VCO $V_{\mathrm{i}}$. The frequency of $\Phi_{\mathrm{i}}$ was set on each time step to $f_{\mathrm{i}}(t)$, as given above. In theory, if $V_{\mathrm{i}}$ is also at frequency $f_{\mathrm{i}}(t)$ at time $t$ and if $V_{\mathrm{i}}$ and $\Phi_{\mathrm{i}}$ are at phase 0 at time 0 , then $\Phi_{\mathrm{i}}$ and $V_{\mathrm{i}}$ will always be at the same phase at any time and we can use the phase of $\Phi_{\mathrm{i}}$ as a measure of the phase of $V_{\mathrm{i}}$. In reality, $V_{\mathrm{i}}$ 's frequency will not be perfectly controllable, so the difference in phase between $\Phi_{\mathrm{i}}$ and $V_{\mathrm{i}}$ is a measure of the phase error $V_{\mathrm{i}}$ has accumulated. We recorded this error (the difference in phases) each time any cell in $V_{\mathrm{i}}$ emitted a spike.

Inaccuracies in $F(I)$ can be particularly noticeable in the phase differences between baseline oscillators because these both maintain a constant frequency, which should be identical. If a network oscillator's frequency is slightly different from the abstract VCO's frequency due to $F(I)$ interpolation, the phase difference will show an apparent linear drifting error that does not actually affect the network model. We avoided this cosmetic problem by specifically selecting the baseline frequency as one of the points of the measured $F(I)$ curve so that no interpolation was needed. This ensured the abstract and network oscillators were truly operating at identical frequencies at baseline.

Model description. Our model is summarized in Tables S1-S7 (parameters in Table 1) following the good model description practices suggested by Nordlie et al. (2009). The architecture of the model is summarized in Figure 2.

Our network oscillatory interference model is composed of a single cell $G$ (the grid cell itself), which receives input from one or more (generally three) oscillatory networks $V_{\mathrm{i}}, 0 \leq i \leq n_{\mathrm{VCO}}$, which are identical apart from their inputs. $V_{0}$ will often be called the baseline network oscillator.

Cells within each network $V_{\mathrm{i}}$ are recurrently coupled all-to-all (no self-connections) by identical synapses or gap junctions of strength $g$ (except in one simulation where the connectivity probability is $p=$ 0.01 and all $V_{\mathrm{i}}$ use the same connectivity matrix for reasons of com- 
Table 1. Default parameters

\begin{tabular}{|c|c|c|}
\hline Name & $\begin{array}{l}\text { Default value } \\
\text { (if not specified) }\end{array}$ & Description \\
\hline$n$ & 1 & Number of cells per oscillator network \\
\hline P & 1 & Connectivity probability in oscillator networks \\
\hline$\sigma$ & 0 & Standard deviation of voltage noise term \\
\hline$g$ & $0.1,256$ & $V_{\mathrm{i}}$ internal gap junction or synaptic conductance, respectively \\
\hline$\tau$ & $4.5 \mathrm{~ms}$ & Time constant of cell $G$ \\
\hline$w_{\mathrm{i}}$ & 0.04 & Synaptic weight from neurons in $V_{i}$ onto $G$ \\
\hline$t_{\text {gate }}$ & $0.04 \mathrm{~s}$ & Duration of baseline gating effect on $G$ \\
\hline$C_{\text {res }}$ & $-0.011 / \mathrm{s}$ & Decay rate in resonate-and-fire model \\
\hline$\omega_{\text {res }}$ & $7 \mathrm{~Hz}$ & Resonant frequency in resonate-and-fire model \\
\hline$\theta$ & 3 & Abstract grid cell model threshold \\
\hline$\omega_{\mathrm{b}}$ & $7 \mathrm{~Hz}$ & Baseline oscillator frequency \\
\hline$\beta$ & $2 \mathrm{~Hz} /(\mathrm{m} / \mathrm{s})$ & $\begin{array}{l}\text { Term giving grid field spacing by relating velocities to } \\
\text { oscillator frequencies }\end{array}$ \\
\hline$C$ & 100 & Capacitance-like term in simple model \\
\hline$v_{\mathrm{r}}$ & -60 & Resting potential in simple model \\
\hline$v_{\mathrm{t}}$ & -40 & Spiking threshold in simple model \\
\hline$v_{\text {peak }}$ & 35 & Action potential peak in simple model \\
\hline$a$ & 0.03 & Recovery variable rate in simple model \\
\hline$b$ & 2 & $v-u$ coupling in simple model \\
\hline$c$ & -50 & Post-spike membrane potential in simple model \\
\hline$d$ & 100 & $\begin{array}{l}\text { Recovery variable increment during an action potential in } \\
\text { simple model }\end{array}$ \\
\hline$k$ & 0.7 & Voltage variable rate in simple model \\
\hline$\tau_{\mathrm{GABA}}$ & $15 \mathrm{~ms}$ & Inhibitory synapse exponential decay time constant \\
\hline$n_{\mathrm{vco}}$ & 0 & $\begin{array}{l}\text { Number of active oscillators in network simulation (not } \\
\text { counting baseline) }\end{array}$ \\
\hline$C_{m}$ & $5 \mu \mathrm{F} / \mathrm{cm}^{2}$ & Membrane capacitance in biophysical model \\
\hline$g_{\mathrm{Na}}$ & $52 \mathrm{mS} / \mathrm{cm}^{2}$ & Sodium current conductance \\
\hline$g_{\mathrm{NaP}}$ & $0.5 \mathrm{mS} / \mathrm{cm}^{2}$ & Persistent sodium current conductance \\
\hline$g_{\mathrm{K}}$ & $11 \mathrm{mS} / \mathrm{cm}^{2}$ & Potassium current conductance \\
\hline$g_{\mathrm{H}}$ & $1.5 \mathrm{mS} / \mathrm{cm}^{2}$ & H current conductance \\
\hline$g_{\mathrm{L}}$ & $0.5 \mathrm{mS} / \mathrm{cm}^{2}$ & Leak current conductance \\
\hline$E_{\mathrm{Na}}$ & $55 \mathrm{mV}$ & Sodium ion reversal potential \\
\hline$E_{\mathrm{K}}$ & $-90 \mathrm{mV}$ & Potassium ion reversal potential \\
\hline$E_{\mathrm{H}}$ & $-20 \mathrm{mV}$ & H current net reversal potential \\
\hline$E_{\mathrm{L}}$ & $-65 \mathrm{mV}$ & Leakage reversal potential \\
\hline$\theta_{\text {spike }}$ & $20 \mathrm{mV}$ & Threshold for biophysical model spike detection \\
\hline
\end{tabular}

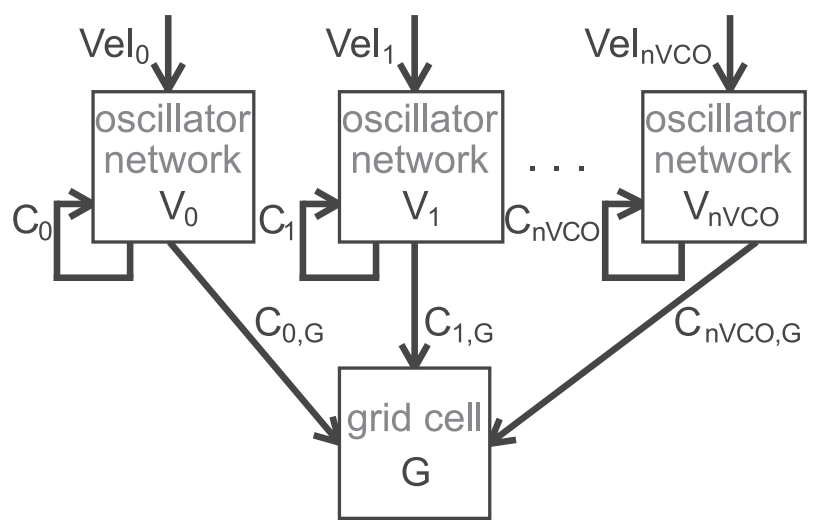

Figure 2. Architecture of the model. The input to the model is a set of $n_{\mathrm{vco}}+1$ velocity signals, $\mathrm{Vel}_{\mathrm{i}}$. These signals go to respective networks $V_{\mathrm{i}}$ that are all-to-all internally coupled over connections $C_{\mathrm{i}}$, which may be either synapses or gap junctions. The grid cell $G$ receives input over synapses $C_{\mathrm{i}, \mathrm{G}}$ from all cells in all $V_{\mathrm{i}}$.

putational efficiency). The cells in each $V_{\mathrm{i}}$ are the only cells that receive external input or noise. All cells in each $V_{\mathrm{i}}$ also project onto $G$ with identical synapses of strength $w_{\mathrm{i}}$. When synapses from all networks have the same strength (i.e., $w_{\mathrm{i}}=w_{\mathrm{j}}$ for all $i, j$ ), the weight is simply referred to as $w$ for conciseness. Importantly, the networks $V_{\mathrm{i}}$ are not coupled to each other, to prevent them from synchronizing with each other (a problem for single-cell oscillatory interference models) (Remme et al., 2009).

$G$ is generally modeled as a leaky integrate-and-fire neuron characterized by its membrane time constant $\tau$. The threshold of the cell is 1 , and after a spike its voltage is reset to 0 . We also considered a modification where oscillator $V_{0}$ is assumed to have a privileged connection onto $G$ such that inputs from any other oscillator $V_{\mathrm{i}}, i \neq 0$, have no effect unless they occur in a $t_{\text {gate }}$ second window after any spike from a cell in $V_{0}$. Thus, a gate that allows inputs from the other $V_{\mathrm{i}}$ networks to reach $G$ 's soma is opened only briefly after the baseline oscillator fires (Ang et al., 2005; Jarsky et al., 2005).

Alternatively, we sometimes modeled $G$ as a resonate-and-fire neuron (Izhikevich, 2001). The model is the simplest model of resonance: the subthreshold dynamics are a two-dimensional linear time-invariant system with a pair of complex conjugate eigenvalues, $c_{\text {res }} \pm \omega_{\text {res }} \sqrt{-1}$ with negative decay rate $\left(c_{\text {res }}<0\right)$ and with resonant frequency $\omega_{\text {res. }}$. The model can be interpreted as a linearization of a resonant biophysical model (Izhikevich, 2001). The 2D state of the neuron can be written as a single complex number. The real part of the neuron's state is thresholded as a spiking mechanism. When the real part of the state crosses from $<1$ to $>1$, a spike is emitted and the state of the neuron is reset to $\sqrt{-1}$. Note that in the original model (Izhikevich, 2001), the imaginary part of the state would be thresholded, which prevents large inputs from immediately causing the cell to fire (but this was desired here).

Finally, for the simulation using inhibitory synapses onto $G$, we modeled $G$ as a simple model (Izhikevich, 2003, 2007) neuron that receives a current injection to fire continuously. The dynamics of the simple model describe a neuron's action potential upstroke and its subthreshold and postspike behavior. The action potential downstroke is modeled explicitly by resetting the membrane potential to a specified value when it reaches a peak value (the peak of an action potential, not a firing threshold). After each spike, the recovery variable is incremented by a fixed amount. This increment represents the build-up of slow currents during the action potential. Our implementation of this model was based on the MATLAB code given in Izhikevich (2007).

Networks $V_{\mathrm{i}}$ are usually modeled as sets of identical simple model neurons with an extra additive voltage noise term. Since discontinuities (like the action potential downstroke) can affect synchronization properties (Teramae and Tanaka, 2004) and other approximations made in the simple model may affect our results, we repeated some of the simulations with a biophysical model.

The biophysical model of an entorhinal cortical layer II stellate cell uses parameters from Acker et al. (2003) but with an additive noise term (instead of their stochastic persistent sodium channel model) and deltacurrent synapses (instead of their AMPA synapse model). This model of entorhinal cortex layer II stellate cells contains standard spiking sodium and potassium channels as well as a noninactivating persistent sodium current and a two-component (fast and slow) H current (Fransén et al., 2004) thought to play a large role in the stellate cell subthreshold dynamics (Magistretti and Alonso, 1999, 2002; Dickson et al., 2000). We decreased the conductance densities in the model (by increasing $C_{\mathrm{m}}$ ) to slow the firing frequencies such that the model could fire at $5 \mathrm{~Hz}$, as needed to match biological noise levels as described below.

All cells in network $V_{\mathrm{i}}$ receive the same scalar input signal $I_{\mathrm{Vel}, \mathrm{i}}(t)$ as a current injection (each network has its own input signal). The magnitude of the input at any time is calculated in terms of the desired population frequency of $V_{\mathrm{i}}$. Given a desired frequency $f_{\mathrm{i}}$, defined above, and the frequency response of the network to a given input, $F\left(I_{\mathrm{Vel}, \mathrm{i}}\right)$, the input to all cells in network $V_{\mathrm{i}}$ is $I_{\mathrm{Vel}, \mathrm{i}}(t)=F^{-1}\left(f_{\mathrm{i}}\right)$.

The primary output measure of the model is the firing of cell $G$. When a single network $V_{0}$ drives $G$ (using $G$ as an indirect measure of the behavior of $V_{0}$ ), the mean $\mu$ and SD $\sigma$ of the interspike intervals (ISIs) of $G$ are measured. In case $G$ fires a burst of spikes in response to sustained inputs, ISIs $<50 \mathrm{~ms}$ long are collapsed into one long ISI, which represents the interval between first spikes of each burst (e.g., consecutive ISIs of length 100, 7, and $90 \mathrm{~ms}$ become a pair of ISIs of 100 and $97 \mathrm{~ms}$ ). This easily allows synchronous population activity in the VCO to be measured. Henceforth, when the mean or variance of the periods of a VCO 
A
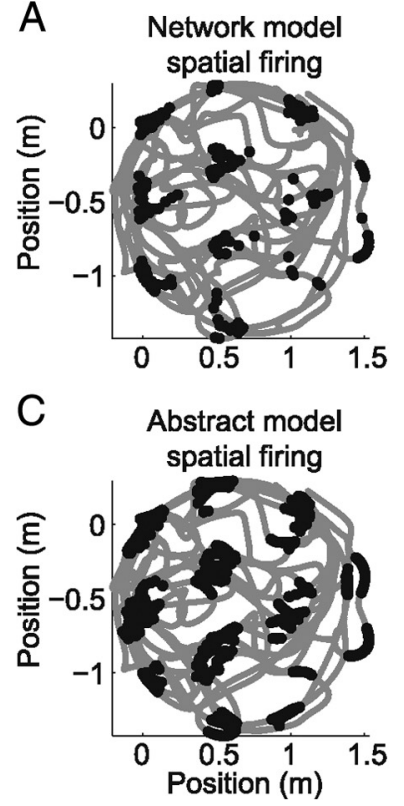

B Network model autocorrelogram

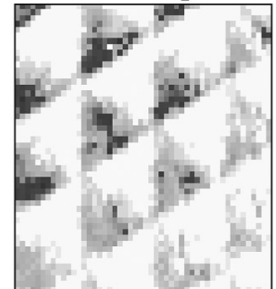

D Abstract model autocorrelogram

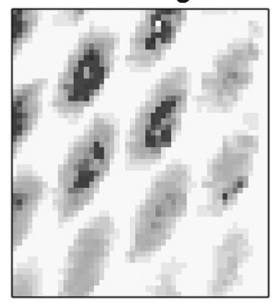

$\mathrm{E}$

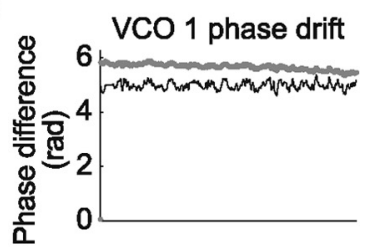

$\mathrm{F}$

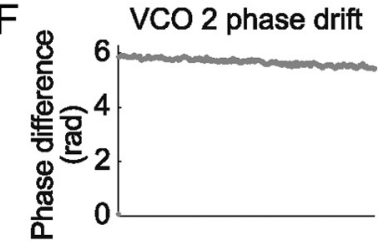

G

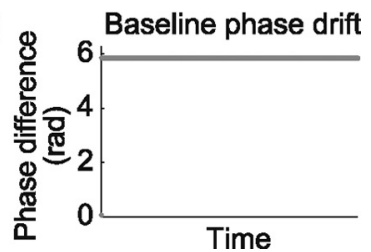

Figure 3. Two-dimensional grid cell with oscillators comprising single, noise-free simple model neurons. $\boldsymbol{A}$, The network receives velocity input corresponding to a smooth two-dimensional trajectory (gray) and a leaky integrator postsynaptic cell generates spikes (black) as output. $\boldsymbol{B}$, The autocorrelogram of the spatial firing in $\boldsymbol{A}$ shows the clear hexagonal pattern characteristic of grid cells. $\boldsymbol{C}, \boldsymbol{D}, A$ simulation of the abstract model produces essentially identical results to $\boldsymbol{A}$ and $\boldsymbol{B}$ apart from spatial displacement and difference in field size. $\boldsymbol{E}, \boldsymbol{F}$, Phase differences between simple model and abstract model of the two active velocity-controlled oscillators show a linear drift over time, and the fluctuations correspond to the velocities along the preferred directions of the respective VCOs. E, Black line, For comparison, the horizontal component of velocity that is encoded by VCO 1. G, The baseline oscillators accumulate no phase error in this noiseless simulation. Parameters: $320 \mathrm{~s} \mathrm{simulation,} n_{\mathrm{VCO}}=2$ (active VCO preferred directions of 0 and $2 \pi / 3$ radians), $n=1, \tau=40 \mathrm{~ms}, w_{0}=0.8, w_{1}=$ $0.14, \omega_{\mathrm{b}}=7.8989 \mathrm{~Hz}$; Table 1 .
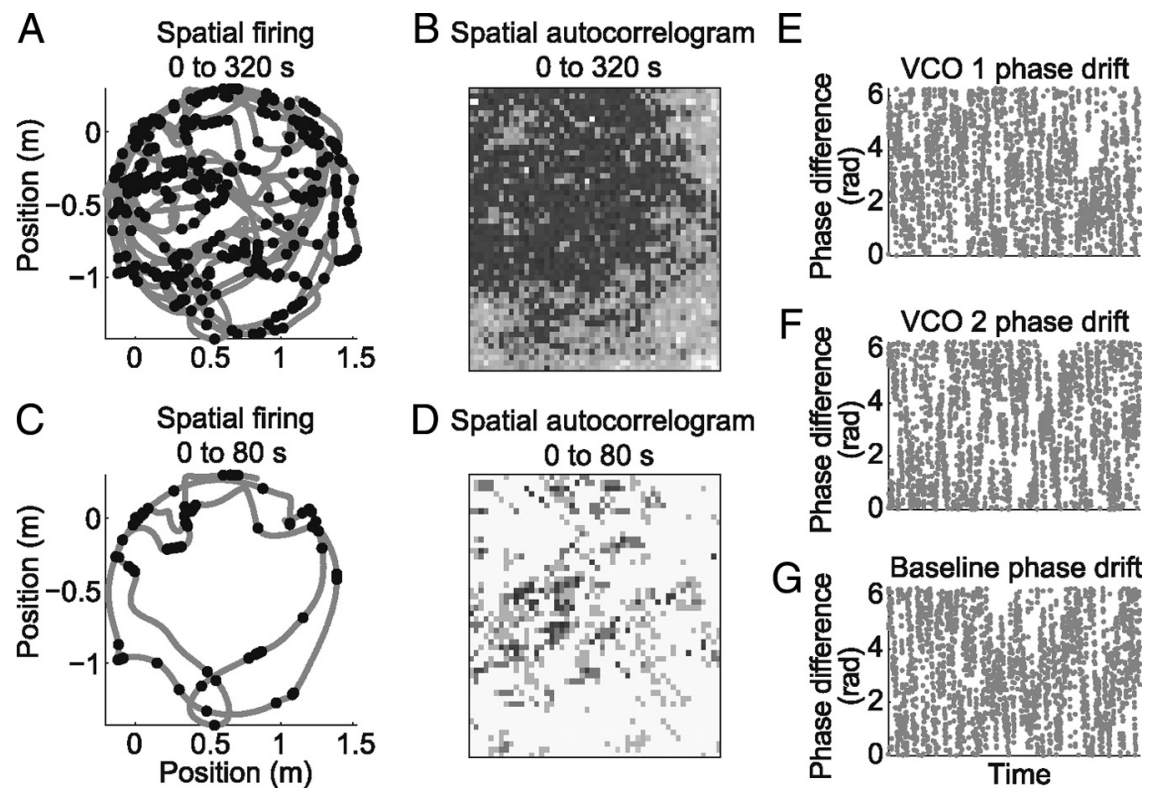

Baseline phase drift

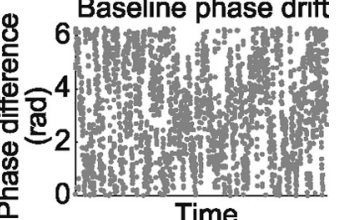

Figure 4. Noisy simple model neurons, when uncoupled, are unfit as oscillators. $A, C$, The network receives velocity input corresponding to a smooth two-dimensional trajectory (gray), and the locations where the simulated grid cell spikes over the entire $320 \mathrm{~s}(\boldsymbol{A})$ and first $80 \mathrm{~s}(\boldsymbol{C})$ are indicated with black dots. $\boldsymbol{B}, \boldsymbol{D}$, The autocorrelogram of the spatial firing shows no clear regularity, even early in the simulation. $\boldsymbol{E}-\boldsymbol{G}$, Phase differences between corresponding oscillators show that realistically noisy neurons lose their correct phases very quickly compared with behavioral timescales. Parameters: $320 \mathrm{~s}$ simulation, $n_{\mathrm{vco}}=2$ [active VCO preferred directions of 0 and $2 \pi / 3$ radians], $n=1, \sigma=100, \tau=40 \mathrm{~ms}, w_{0}=0.8, w_{1}=0.14, \omega_{\mathrm{b}}=$ $7.8989 \mathrm{~Hz}$; Table 1.

zzare referred to, we mean the corresponding statistic of the spikes emitted by G. We can use these statistics to evaluate how well oscillators will perform in the oscillatory interference model. Zilli et al. (2009) derived an expression for the estimated stability of a grid pattern as a function of the mean and variance of the periods of a noisy oscillator. Using the approximation that noise results in oscillator period durations independently drawn from a normal distribution with mean $\mu$ and SD $\sigma$ (in seconds), the estimated stability time formula $t=$ $5 \mu^{3} /(4 \pi \sigma)^{2}$ gives the number of seconds until a grid cell is no longer likely encoding the correct spatial location. This is derived by noting that the difference in lengths of periods of two identical but noisy oscillators at the same frequency has twice the variance $2 \sigma^{2}$ of the length of a period of either oscillator, and the difference in lengths of $n$ consecutive (independent) periods has the variance $2 n \sigma^{2}$. The variance is converted from units of seconds ${ }^{2}$ to radians ${ }^{2}$ $\left(2 n \sigma^{2}(2 \pi)^{2} / \mu^{2}\right.$ radians $\left.^{2}\right)$ and the estimated stability time is the amount of time for the variance to hit a threshold of 2.5 radians $^{2}$ (for further details, see Zilli et al., 2009).

When two or more networks $V_{\mathrm{i}}$ drive $G$, the inputs to $V_{\mathrm{i}}$ represent a spatial trajectory, and a primary measure is the set of spatial positions where $G$ emits spikes. The spatial autocorrelation of the firing positions was calculated numerically in MATLAB by binning the spike positions using the hist 3 function, then calculating the autocorrelation by taking the twodimensional convolution of the histogram with itself (using the conv2 function with the same option). A final measure commonly used is the phase difference between a network oscillator $V_{\mathrm{i}}$ and the corresponding abstract, idealized oscillator $\Phi_{\mathrm{i}}$, as described above.

The parameters of the model are summarized in Table 1, where default values are given (where a simulation uses a different value, it is given in the text or figure caption). The parameters of the simple model neurons $\left(C, v_{\mathrm{r}}, v_{\mathrm{t}}\right.$, $\left.v_{\text {peak }}, a, b, c, d, k\right)$ correspond to a resonant regular spiking neuron (e.g., a cortical pyramidal cell), as given in Izhikevich (2007) (the original model had $b<0$, which we negated to get a resonant model). The biophysical model parameters were taken from Acker et al. (2003), with $C_{\mathrm{m}}$ increased as shorthand for proportionally decreasing all ionic conductances. The noise level $\sigma$ was selected to phenomenologically match experimental data (supplemental material). As the noise $\sigma$ increases, the number of cells $n$ may need to increase and coupling strength $g$ may need to change to maintain synchronization. The baseline frequency $\omega_{\mathrm{b}}$ was set to a frequency near the middle of the measured $F(I)$ curve at a current value where the exact frequency is known. For the LIF model, the time constant $\tau$ and synaptic strength $w$ (and $t_{\text {base }}$ when used) were adjusted to ensure all VCOs fired at nearly the same time, which is need in order for $G$ to fire, and there was a range of $\tau$ and $w$ that produced similar results (possibly changing the size of the fields themselves, up to the point of firing oc- 
curring at all or no locations). For the resonateand-fire model, the decay rate $c_{\text {res }}$ was selected similarly, and the resonant frequency was $\omega_{\text {res }}=\omega_{\mathrm{b}}$. Note that when $c_{\text {res }} \ll 0$, the cell is essentially nonresonant, so we kept $c_{\text {res }} \approx 0$ to focus on the case where resonance may play a large role. The velocity-to-frequency slope $\beta$ controls grid spacing as well as the range of frequencies of the $F(I)$ curve that must be calculated. We used $\beta=2 \mathrm{~Hz} /(\mathrm{m} / \mathrm{s})$, the mean value of $\beta$ for theta frequency oscillations in entorhinal cortex layer II stellate cells (Jeewajee et al., 2008).

Initial suggestions linked grid cells to the membrane resonance of entorhinal cortex layer II stellate cells (Giocomo et al., 2007). Both our biophysical and simple model parameterizations are also resonant; however, as no current evidence specifically implicates entorhinal cortex layer II stellate cells (or resonance in general) in the generation of the grid pattern, preliminary simulations of many of these results were also tested with the networks $V_{\mathrm{i}}$ comprising simple model neurons parameterized as integrators instead of resonators (setting $b=-2$ ) and all tested results were reproducible with that model. Generally, both types of neurons are found in all regions. Resonant cells in the medial temporal lobe also include, for instance, CA1 pyramidals (Leung and $\mathrm{Yu}, 1998$; Hu et al., 2002, 2009) and interneurons (Chapman and Lacaille, 1999), cells in lateral and basolateral amygdala (Pape et al.,1998), and $>80 \%$ of layer II parasubicular neurons (including principal stellate and pyramidal neurons in addition to interneurons) (Glasgow and Chapman, 2007). Cells reported to be nonresonant (integrators) include nonstellate cells in entorhinal cortex layer II (Alonso and Klink, 1993; Haas and White, 2002; Erchova et al., 2004) and pyramidal cells in entorhinal cortex layer III (Erchova et al., 2004). However, conclusions regarding resonance drawn from in vitro experiments may not always correspond to in vivo behavior (Fernandez and White, 2008; Prescott et al., 2008).

\section{Results}

Oscillatory interference

Our oscillatory interference model was composed of one baseline oscillator $V_{0}$, which maintained a constant frequency and a number of active oscillators $V_{\mathrm{i}}, \mathrm{i} \geq$ 1 , which changed their phase relative to the baseline oscillator to encode positional information. The simulated animal's body velocity input changed the oscillators' frequencies slightly above or below the baseline frequency such that the set of locations where any single active oscillator was in phase with the baseline oscillator formed a set of parallel bands in the environment (though these bands do not appear as spiking in this model).

The function of the model is easily demonstrated using the abstract version
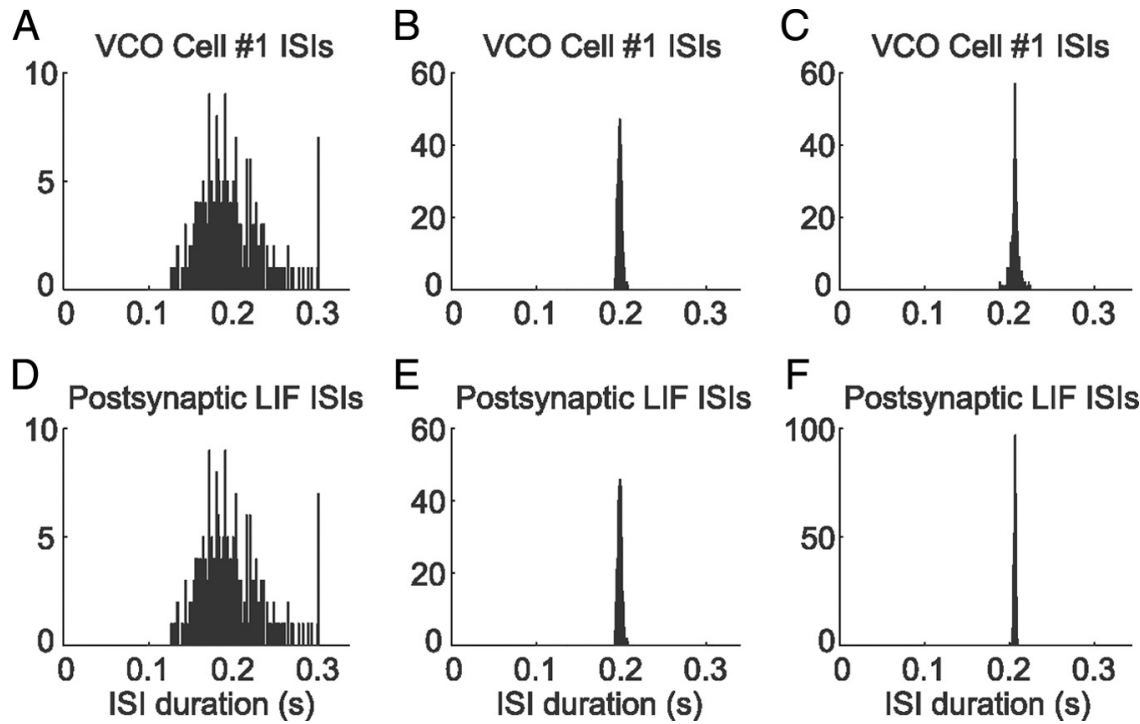

Figure 5. ISI histograms for cells in three conditions. $\boldsymbol{A}$, ISIs of an uncoupled, noisy simple model neuron are much more variable than when multiple cells are coupled (as in $\boldsymbol{B}$ and $\boldsymbol{C}$ ). $\boldsymbol{B}$, ISIs of one noisy neuron from a network of 250 gapjunction-coupled neurons. C, ISIs of one noisy neuron from a network of 250 synaptically coupled neurons. D, ISIs of a LIF cell driven by the cell in $\boldsymbol{A}$. The histograms are identical, showing that the postsynaptic cell directly inherits the variability of the cell driving it. $\boldsymbol{E}$, ISIs of an LIF cell receiving input from all 250 gap-junction-coupled neurons. The variability of individual gap-junction-coupled neurons is very similar to the variability of the postsynaptic cell. $\boldsymbol{F}$, ISIs of an LIF cell receiving input from all 250 synaptically coupled neurons. The individual cells $(\boldsymbol{C})$ are noticeably more variable than the postsynaptic cell (and see Fig. 8 for a more extreme case). Parameters: 60 s simulations, $n=1$ ( $w=1.2$ ) or $n=250$ ( $w=$ 0.0048), $\sigma=100$; Table 1.
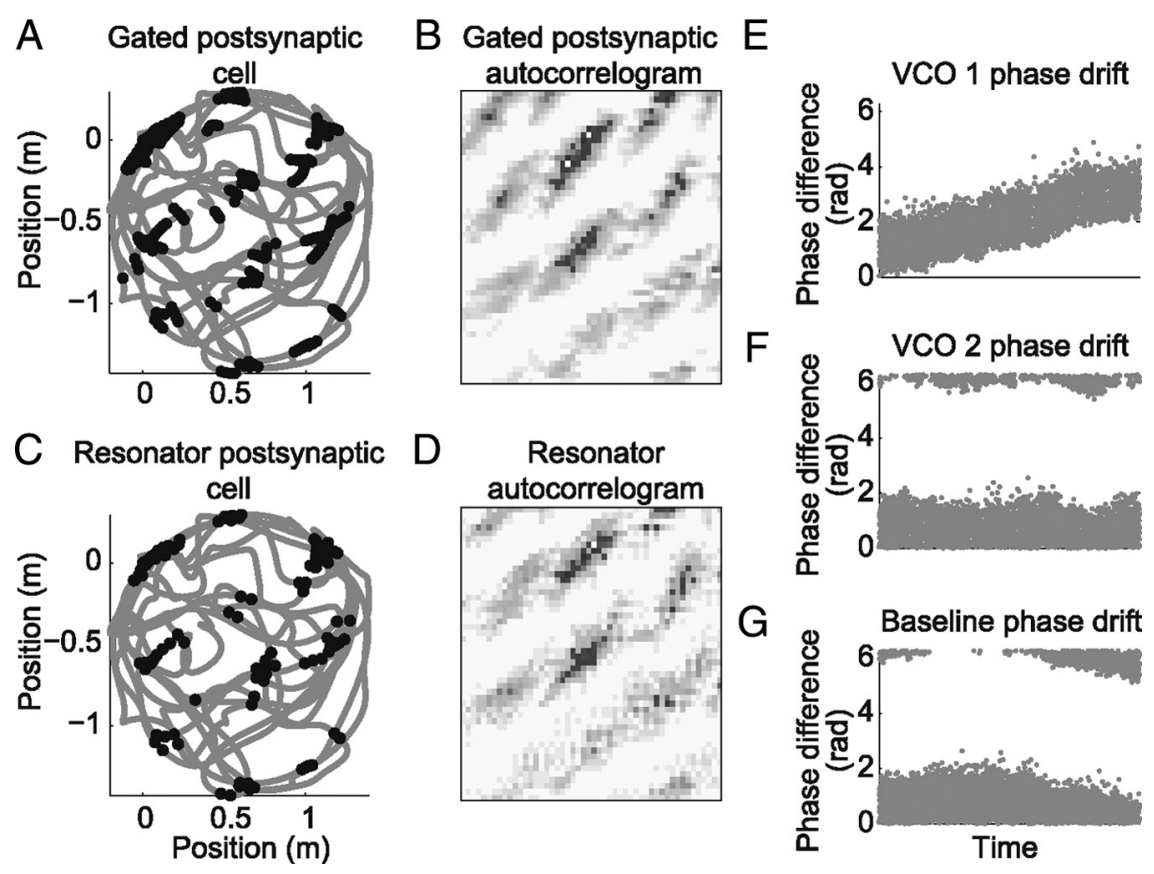

Figure 6. Two-dimensional grid cell with network oscillators made of noisy, synaptically coupled simple model neurons. $\boldsymbol{A}$, The network receives velocity input corresponding to a smooth two-dimensional trajectory (gray) and a gated, leaky integrator postsynaptic cell generates spikes (black). $\boldsymbol{B}$, The autocorrelogram of the spatial firing in $\boldsymbol{A}$ shows hexagonal periodicity. Parameters: $t_{\text {gate }}=0.001 \mathrm{~s}, \tau=5 \mathrm{~ms}, w=0.0016$. C, $D$, The spatial firing and spatial autocorrelogram of the same network but with a resonant postsynaptic cell. Parameters: $c_{\text {res }}=-0.01, \omega_{\text {res }}=\omega_{b}, w_{0}=0.0024, w_{1}=w_{2}=0.002$. E-G, Phase differences between network and abstract models of the two active velocity-controlled oscillators. The average of the phase differences remains relatively constant, although individual cells are firing over a much wider range of phases compared with when the cells are instead gap-junction-coupled. General parameters: 280 s simulation. $n_{\mathrm{Vco}}=2$ [active VCO preferred directions of 0 and $2 \pi / 3$ radians], $n=250, \sigma=100, \omega_{\mathrm{b}}=7.2543 \mathrm{~Hz}$; Table 1 . 

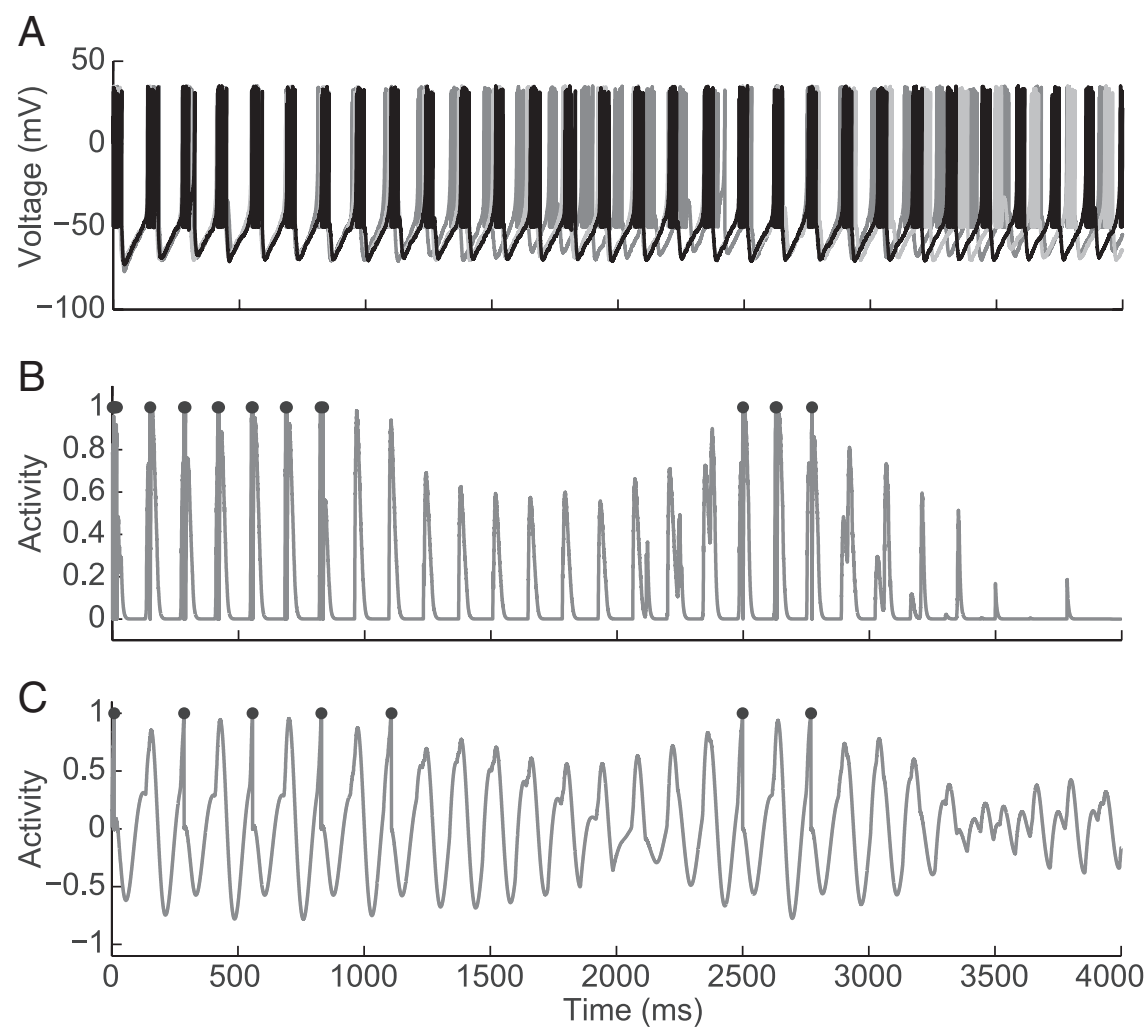

Figure 7. Combining the activity of multiple synaptically coupled oscillators. $A$, Voltage traces of single neurons from $V_{0}($ black), $V_{1}$ (light gray), and $V_{2}$ (dark gray) in the first $4 \mathrm{~s}$ of the trajectory shown in Figure $6 . \boldsymbol{B}$, The neurons in $\boldsymbol{A}$ project onto an integrate-and-fire neuron where cells in $V_{0}$ do not produce activity, but instead open a gate allowing cells in $V_{1}$ and $V_{2}$ to produce depolarization and sometimes spikes (black). Parameters: $t_{\text {gate }}=0.001 \mathrm{~s}, \tau=5 \mathrm{~ms}, w=0.0016$. C, The neurons in $A$ project onto a resonate-and-fire neuron with resonant frequency equal to the baseline frequency. Parameters: $c_{\text {res }}=-0.01, \omega_{\text {res }}=$ $\omega_{\mathrm{b}}, w_{0}=0.0024, w_{1}=w_{2}=0.002$. General parameters: $n_{\mathrm{VCO}}=2$ (active VCO preferred directions of 0 and $2 \pi / 3$ radians), $n=250, \sigma=100, \omega_{\mathrm{b}}=7.2543 \mathrm{~Hz}$; Table 1 .

(Burgess et al., 2007), where the VCO phases evolve according to

$$
d \varphi_{\mathrm{i}} / d t=2 \pi \omega_{\mathrm{b}}+2 \pi \beta s(t) \cos \left[\phi_{\mathrm{i}}-\phi(t)\right],(i \geq 1)
$$

and

$$
d \varphi_{0} / d t=2 \pi \omega_{\mathrm{b}} .
$$

We define $f_{\mathrm{i}}(t)=\omega_{\mathrm{b}}+\beta s(t) \cos \left[\phi_{\mathrm{i}}-\phi(t)\right]$, the desired frequency of oscillator $i$ at time $t$, as a function of velocity at time $t(s$ and $\phi$ ) and preferred direction $\phi_{\mathrm{i}}$. Notice in Equations 1 and 2 that the phase difference $\varphi_{\mathrm{i}}-\varphi_{0}$ changes at frequency $\beta s(t) \cos \left[\phi_{\mathrm{i}}-\right.$ $\phi(t)] \mathrm{Hz}$. If the animal moves in direction $\phi_{\mathrm{i}}$ at $s(t)=1 \mathrm{~m} / \mathrm{s}$ and if $\beta=1 \mathrm{~Hz} /(\mathrm{m} / \mathrm{s})$, the phase difference will oscillate at frequency $\beta s(t)=1 \mathrm{~Hz}$. If the phase difference is initially 0 , the phase difference will return to zero each $1 \mathrm{~m}$ the animal walks (like a digit in an odometer rolling over). If the animal were to move at $s(t)=$ $2 \mathrm{~m} / \mathrm{s}$ then the phase difference would oscillate at $\beta s(t)=2 \mathrm{~Hz}$ and so the phase difference will still return to zero each $1 \mathrm{~m}$ the animal walks. In fact, this is true for any $1 \mathrm{D}$ trajectory $s(t)$. If $\beta=$ $2 \mathrm{~Hz} /(\mathrm{m} / \mathrm{s})$, then the phase difference will return to zero each $1 / 2 \mathrm{~m}$ (generally each $1 / \beta \mathrm{m}$ ), so $\beta$ acts to set the distance in space over which the phase difference repeats.

The abstract grid cell is then said to fire if $\Sigma_{1 \leq i \leq n \mathrm{VCO}}\left(\cos \varphi_{0}+\right.$ $\cos \varphi_{\mathrm{i}}$ ) exceeds a threshold $\theta$. Each term in the sum represents the readout via an interference mechanism of the respective phase differences, and the model fires when all of these phase differences are near 0 . When the directions $\phi_{\mathrm{i}}$ are separated by $60^{\circ}$ increments (and are not all colinear), the phase differences all equal 0 at positions that are hexagonally arrayed.

Three neighboring fields form an equilateral triangle where the distance from the base of the triangle to the opposite vertex is $1 / \beta \mathrm{m}$. By trigonometry, the distance between neighboring fields is then $\lambda=$ $2 /(\sqrt{ } 3 \beta) \mathrm{m}$. This model is thoroughly described in a number of previous publications (Burgess et al., 2007; Giocomo et al., 2007; Burgess, 2008; Hasselmo, 2008) and further details can be found there.

\section{Single cell oscillators}

In Figure 3, noiseless simple model neurons are used as single-cell VCOs (Burgess, 2008). For computational efficiency, this manuscript focuses on the case of two active VCOs at angles $0^{\circ}$ and $120^{\circ}$, although preliminary simulations suggest our model is successful when $n_{\mathrm{VCO}}=4$ (three active VCOs at angles $0^{\circ}, 120^{\circ}$, and $240^{\circ}$ ). On each time step of the simulation, the instantaneous velocity inputed along the directions $0^{\circ}$ and $120^{\circ}$ set the frequency of the active oscillators (via the $F(I)$ curve) (Fig. $1 A$ ) so that the phase difference changed to represent the distance traveled during that time step. At the positions where the oscillators were all in phase, the postsynaptic cell $G$ was receiving maximal input.

In Figure 3, input from the baseline oscillator neuron $V_{0}$ is made particularly strong and the inputs from the two active oscillators $V_{1}$ and $V_{2}$ are scaled so that a single input is subthreshold and both inputs must occur shortly after the baseline input to push $G$ over threshold. If all synaptic weights were equal, $G$ would produce either tiny or asteriskshaped fields (Burgess, 2008, his Figs. 7 and 8) with lines extending along directions where subsets of VCOs are in phase. Burgess (2008) used a multiplicative baseline sinusoid to solve this problem, and our comparatively stronger baseline input is an alternative solution. With the strong baseline, the firing fields of the grids become triangular in shape, as seen in Figure 3, $A$ and $B$.

Despite the unusual shape, the hexagonal spatial pattern compares well to the output of the abstract version of the model shown in Figure 3, $C$ and $D$. The phase error between respective neural and abstract oscillators, a quantitative measure of performance, is plotted in Figure 3, $E-G$. The near-flatness of the lines shows that the neural model here is performing almost perfectly. The slight fluctuations in phase errors of a VCO are closely related to its velocity input, as shown by the superimposed velocity signal in Figure 3E. These fluctuations are much larger when the input is an unsmoothed spatial trajectory (supplemental Fig. S1, available at www.jneurosci.org as supplemental material).

The similarity of the phase difference error to the velocity input suggests a relationship between changes in the input and changes in the phase error. In fact, it is a general property of neurons that changes in input produce transient, historydependent changes in the $F(I)$ relation (supplemental text and supplemental Figs. S2, S3), which we refer to as adaptation. For 
example, changing $I$ to a level at which the neuron should fire at $7 \mathrm{~Hz}$ actually results in a brief period of slightly higher or lower frequency, which will introduce a small phase error. This error can be minimized by reducing $\beta$ to reduce the range of $\mathrm{VCO}$ frequencies. However, decreasing $\beta$ will also increase the relative impact of noiserelated VCO frequency fluctuations. Additionally, the grid spacing $\lambda=2 /(\sqrt{ } 3 \beta)$ is a function only of $\beta$, as shown above, so the spacing cannot be held constant as $\beta$ is varied, meaning these opposing pressures on $\beta$ directly correspond to pressures on grid spacing.

On the single cell level, however, the effects of noise are far greater than the effects of adaptation. A simulation using single, noisy cells as oscillators (Fig. 4) completely failed to produce the grid pattern. The intrinsic noise level used in this and subsequent simulations was selected (see supplemental material) so that an uncoupled simulated cell had the same ISI mean and variance as the persistent spiking cell with the median estimated stability time from Zilli et al. (2009). An example ISI histogram of a simple model cell with this noise level is shown in Figure $5 A$. From the phase errors in Figure 4, $E-G$, it is clear that the oscillators lose their correct phase relative to the baseline almost immediately. This result was expected on the basis of previous noisy simulations (Burgess et al., 2007; Giocomo and Hasselmo, 2008) and is consistent with the estimated time scale for the level of noise used (Zilli et al., 2009).

\section{Network oscillators}

Because coupling can reduce the period variance of noisy oscillators as a network (Needleman et al., 2001) and individually (Ly and Ermentrout, 2010), we investigated whether networks of coupled, noisy oscillators could play the role of VCOs. For both synaptic and gap-junction coupling, we found that $n=250$ all-to-all connected, noisy, simple model cells (coupling strengths $g=256$ and $g=0.1$, respectively) produced synchronized populations with acceptably low period variance of the network as a whole and the individual units (for the effects of varying important parameters, see supplemental material and supplemental Fig. S4, available at www.jneurosci. org as supplemental material). We can measure the $F(I)$ curves of these networks (Fig. 1), then drive the network to fire at any desired frequency, just as with the single cell VCOs.

Three synaptically coupled networks, one acting as a baseline oscillator, together produce the simulation output shown in Figure 6. A voltage trace of one neuron from each $V_{\mathrm{i}}$ is shown in Figure 7 , along with the activity of the gated LIF cell simulated in Figure 6, $A$ and $B$, and a resonate-and-fire cell shown in Figure 6, $C$ and $D$. Both types of postsynaptic cell produced reasonable hexagonally arrayed firing. The blurring directed along the preferred direction of $V_{2}$ is consistent with the phase errors in $V_{1}$
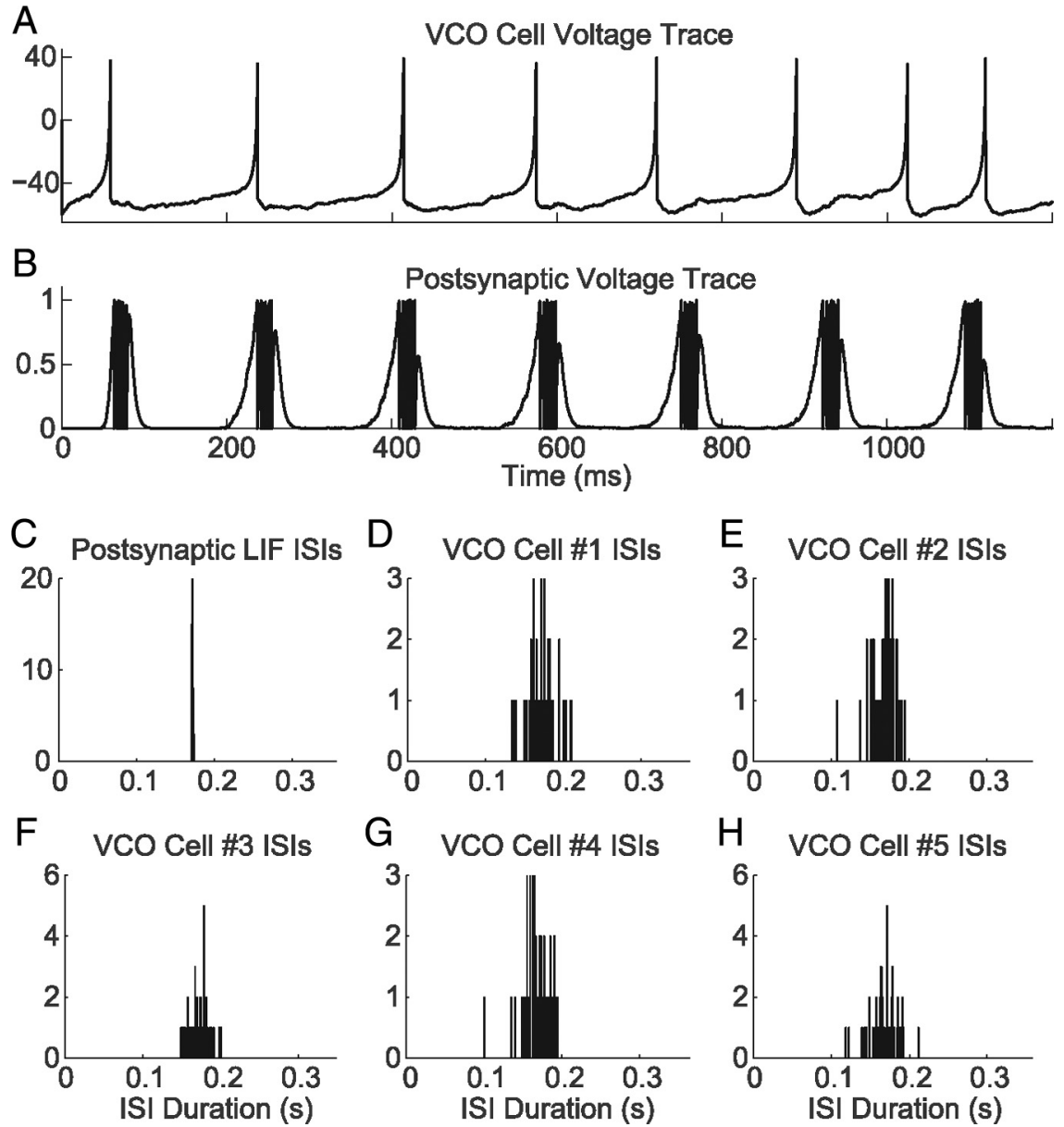

Figure 8. A large network oscillator with a low connection probability can produce low-variance network oscillations while individual cells remain highly variable. $\boldsymbol{A}$, Voltage trace over a short period of time of one of 5000 cells in a network where there is probability that any cell X connects to any other cell Y. The firing is irregular and the effects of noise are clear. $\boldsymbol{B}$, Activity of postsyapticintegrate-and-fire cell receiving input from all 5000 oscillator cells including the one in $\boldsymbol{A}$. The inputs to the postsynare fairly spread out in time during each period so the cell fires in a burst. Comparing $\boldsymbol{A}$ to $\boldsymbol{B}$, it can be seen that an

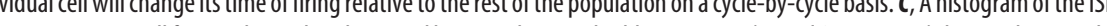
5000 cells was $0.015 \mathrm{~s}, \sim 20$ times that of the postsynaptic cell in $\boldsymbol{B}$ and approximately half of the period SD of the uncoupled cells. Parameters: 10 s simulations, $I=95.8, n=5000$ cells, $w=0.0006,1 \%$ connectivity; Table 1 .

(Fig. $6 E$ ) but not $V_{0}$ and $V_{2}$ (Fig. $6 F, G$ ) as expected from previous analysis (Zilli et al., 2009). This simulation using synaptically coupled cells is similar to that of Hasselmo (2008), where the persistent spiking input was assumed to come in population bursts, shown here in Figure 7 . Here the bursts are caused by strong synaptic coupling, as the neural model does not burst when uncoupled (supplemental Fig. S5, available at www. jneurosci.org as supplemental material) or when less tightly synchronized (Fig. $8 \mathrm{~A}$ ).

Instead of using synaptic connections, successful performance also occurs if the cells in the VCOs are coupled through gap junctions. Supplemental Figure S6 (available at www.jneurosci. org as supplemental material) shows the same simulation but with gap-junction coupling instead of synaptic coupling, and a voltage trace of one cell from each $V_{\mathrm{i}}$ and $G$ are plotted in supplemental Figure S7 (available at www.jneurosci.org as supplemental material). Achieving large fields proved difficult with the resonate-and-fire model with a low damping constant, but was successful with the gated, integrate-and-fire cell. When gap- 

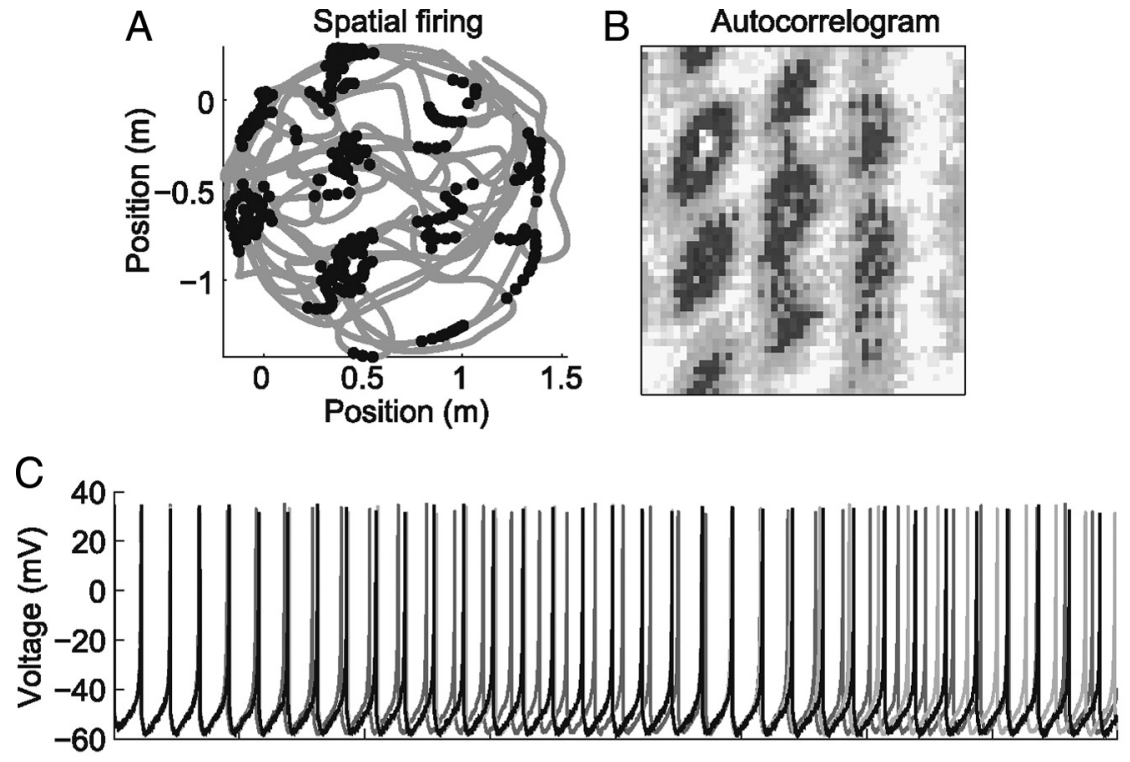

D

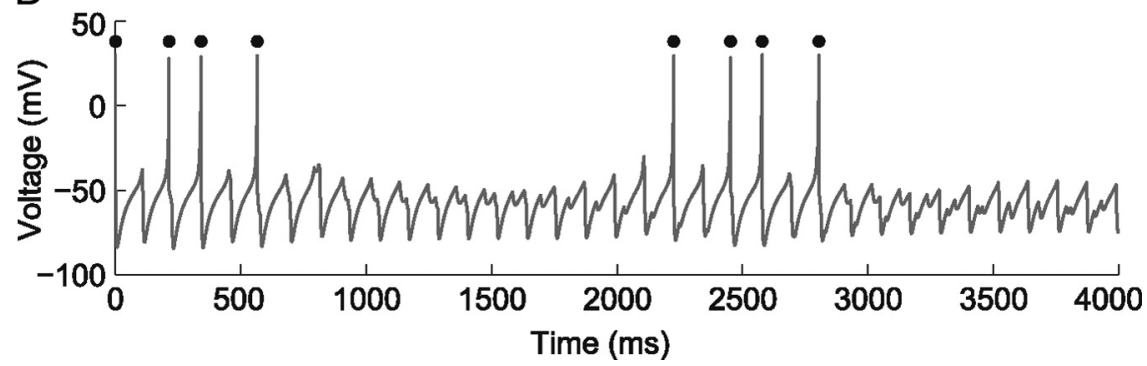

Figure 9. Inhibitory noisy simple model neurons as oscillators. $\boldsymbol{A}$, The network receives velocity input corresponding to a smooth two-dimensional trajectory (gray) and a spontaneously spiking simple model postsynaptic cell generates spikes (black). $\boldsymbol{B}$, The autocorrelogram of the spatial firing in $\boldsymbol{A}$ demonstrates clear hexagonal periodicity. $\boldsymbol{C}$, Voltage traces of single cells from gap-junction-coupled networks $V_{0}$ (light gray), $V_{1}$ (dark gray), and $V_{2}$ (black). $\boldsymbol{D}$, The cells in each $V_{i}$ project with inhibitory synapses onto a simple model cell firing at $\omega_{\mathrm{b}}$. When the $V_{\mathrm{i}}$ are out of phase, the cell is tonically inhibited, but when the $V_{\mathrm{i}}$ move into phase, there is enough time for the cell to fire between volleys of inhibition. Parameters: $320 \mathrm{~s} \mathrm{simulation,} n_{\mathrm{vco}}=2$ (active VCO preferred directions of 0 and $2 \pi / 3$ radians), $n=250, \sigma=100, \omega_{\mathrm{b}}=8.9735 \mathrm{~Hz}, w_{0}=-3.2, w_{1}=w_{2}=-0.76$; Table 1 .

In physiological experiments, it is usually only feasible to record from very few cells at any time, so it is worth considering how the ISI statistics of individual cells in a synchronized VCO network relate to the ISI statistics of the network itself in these simulations. In Figure 5, ISI histograms are shown for an uncoupled cell compared with cells from coupled networks and the variability-reducing effect of synchronization is particularly clear (Fig. 8). The simulated histogram in Figure $5 A$ is comparable to the experimental subthreshold oscillation period histograms in Giocomo and Hasselmo's (2008) supplemental Figure S5A (available at www. jneurosci.org as supplemental material). For synchronized gap-junction-coupled neurons, Figure $5, B$ and $E$, individual neurons had essentially identical statistics to the network as a whole. When neurons were synaptically connected, individual neurons also tended to have similar periods to the network as a whole, but individual cells had a higher variability (larger ISI $\mathrm{SD}$ ) (Fig. 5C,F). However, if the level of connectivity in the network is much lower, the network as a whole can produce low-variance oscillations even though the individual cells in the network show considerable ISI variance (Fig. 8). These sparsely coupled oscillators can still be used successfully in the model (Fig. 11). Thus, the observation of irregular firing in vivo does not rule out highly regular population-level oscillations.

However, coupling is not helpful against all forms of noise. With as little as $5 \%$ of the total noise variance correlated across all cells in one network, prelimi-

junction coupled, the cells did not burst and the smaller range of spiking phases (compared with synaptically coupled cells) may have a role in the increased difficulty we found in achieving larger fields.

The lack of excitatory recurrent connections in entorhinal cortex layer II (Dhillon and Jones, 2000; Couey and Witter, 2010) suggests that the oscillators may be inhibitory interneurons (if the oscillators are located there). To test this theory, we modeled the $V_{\mathrm{i}}$ neurons as gap-junction-coupled inhibitory neurons and $G$ as a simple model neuron driven to fire at the baseline frequency. The inhibition from $V_{\mathrm{i}}$ onto $G$ is modeled as an exponentially decaying input. As shown in Figure 9, when the VCOs are out of phase, $G$ is tonically inhibited, but when the VCOs are in phase (in a grid field) there is a long enough window between the inhibitory inputs that the cell can fire, and it produces the characteristic field spacing as a result.

Though the simple model is an excellent approximation of the subthreshold dynamics of biophysical models, it is worth verifying our results with a relatively more realistic model. In Figure 10, we show the results of a simulation where the VCO networks are now composed of noisy biophysical neurons (with currents $I_{\mathrm{Na}}$, $I_{\mathrm{K}}, I_{\mathrm{NaP}}, I_{\mathrm{H} \text { fast }}$, and $\left.I_{\mathrm{H} \text { slow }}\right)$. The output of this simulation is at least as good as the previous simulations. nary simulations of a network of 250 gap-junction-coupled simple model neurons showed an order of magnitude decrease in stability times compared with the case of each cell receiving the same total level of fully independent noise. In fact, a network of 2500 gap-junction-coupled simple model neurons with $5 \%$ of the total noise variance correlated (and 95\% independent) has approximately the same variability as a single cell with a noise variance at only $5 \%$ of $\sigma^{2}$, the value matched to the experimental data, clearly demonstrating the inability of coupling to correct this type of noise. We used independent noise sources in our simulations to avoid this problem, although realistically there is likely to be some unknown degree of correlation. This problem is an important point for future work, as it could potentially be a fundamental flaw with this approach.

Overall, our simulations suggest that a variety of realistic mechanisms can implement an oscillatory interference model, despite large amounts of independent noise in individual oscillators.

\section{Discussion}

We demonstrated that neural oscillators with realistic levels of noise can be coupled to produce network oscillations with less variability than the individual, uncoupled oscillators and that the 

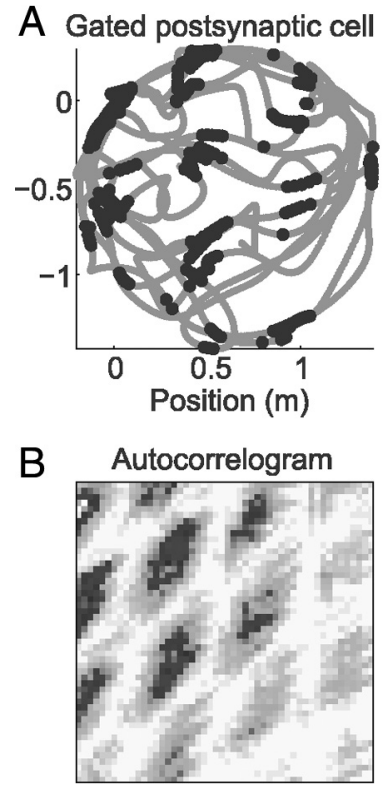

\section{C}
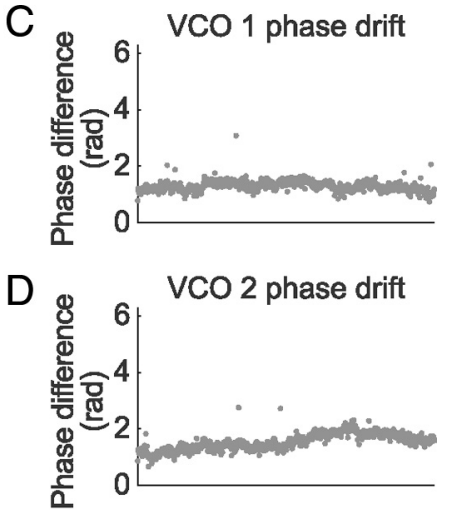

E

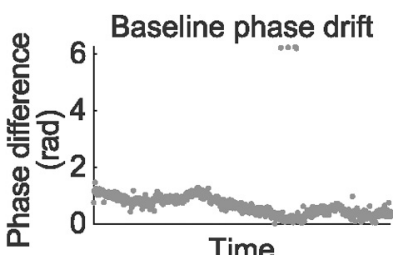

Figure 10. Noisy biophysical neuron networks (synaptically coupled) produce usable velocity controlled oscillators. $\boldsymbol{A}$, The grid cell is modeled as a gated, leaky integrate-and-fire cell and its spiking (black) is shown along a $2 \mathrm{D}$ trajectory (gray). $\boldsymbol{B}$, The autocorrelogram of the spatial firing in $\boldsymbol{A}$ confirms the clear hexagonal periodicity. $\mathbf{C}-\boldsymbol{E}$, The phase differences for all oscillators remain fairly steady during the simulation, demonstrating that the biophysical model is capable of implementing the oscillatory interference mechanism. Parameters: 240 s simulation. $n_{\mathrm{vco}}=$ 2 (active VCO preferred directions of 0 and $2 \pi / 3$ radians), $n=250, g=80, \sigma=3.44, t_{\text {gate }}=$ $0.040 \mathrm{~s}, \tau=50 \mathrm{~ms}, w=0.0024, \omega_{\mathrm{b}}=8.9519 \mathrm{~Hz}$; Table 1 .
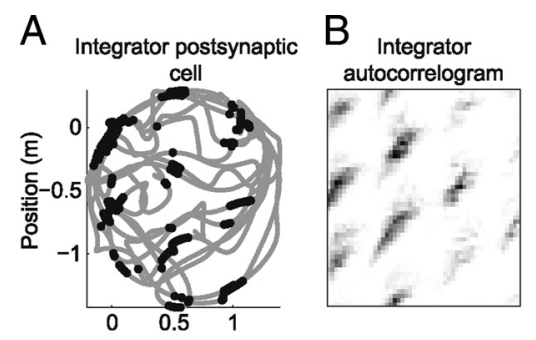

E
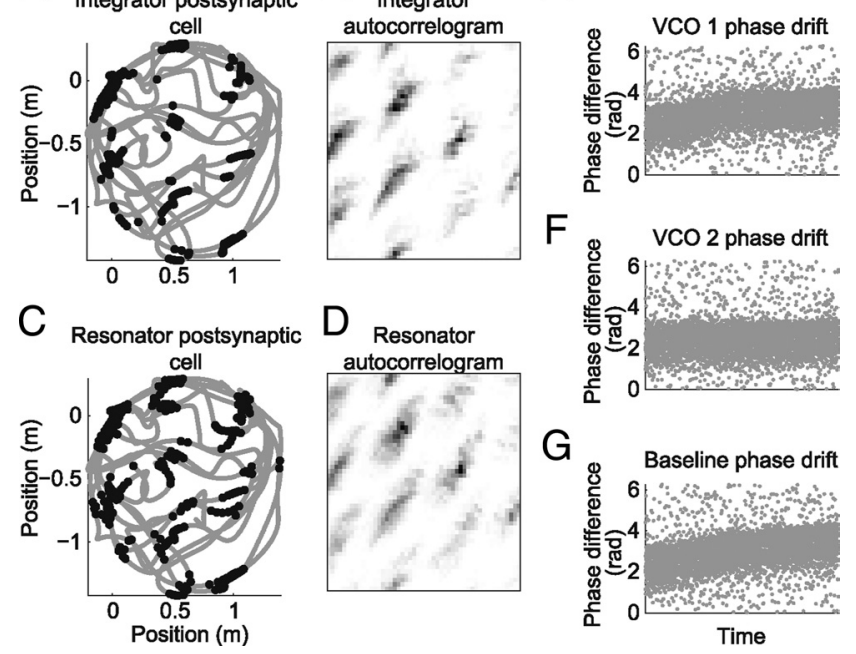

Figure 11. Sparsely coupled simple model neuron networks as oscillators. $A$, The spatial trajectory (gray) provides the input to three oscillator networks of $n=5000$ neurons with a $1 \%$ connectivity probability. Though the individual cells in the networks are highly variable (Fig. 8), the network-level variability is low so the integrate-and-fire postsynaptic cell spikes (black) in a stable grid pattern during the 240 s. Parameters: $\tau=12 \mathrm{ms,}$ $w_{0}=0.00019, w_{1}=w_{2}=0.00018$. $\boldsymbol{B}$, Spatial autocorrelogram of the spiking in $\boldsymbol{A}$. C, The resonate-and-fire postsynaptic cell is also able to combine its inputs to produce a stable grid. Parameters: $c_{\text {res }}=-0.01, w_{\text {res }}=\omega_{b}, w=0.001$. D, Spatial autocorrelogram of the spiking in C. E-G, The highly variable individual oscillators spike over most phases, but the average phase of the network shows very little phase drift compared with the abstract model. Every 25 th phase difference is plotted due to the large number of spikes. Parameters: 240 s simulation. $n_{\mathrm{vco}}=2$ [active VCO preferred directions of 0 and $2 \pi / 3$ radians], $n=5000, p=0.01, g=256, \sigma=100, \omega_{\mathrm{b}}=8.7942 \mathrm{~Hz}$; Table 1. individual oscillators in a network may or may not show this reduction in variance. We then showed that the frequency of such an oscillatory network can be controlled to an acceptable but not perfect level of accuracy. Finally, multiple such oscillatory networks can be combined to produce grid cells with spatial firing that is relatively stable on behavioral timescales. In particular, the oscillator networks may be either gap-junction or synaptically coupled and both excitatory and inhibitory oscillator neurons can be used. We also showed that stable two-dimensional grids can be generated using biophysical model neurons, suggesting a wide range of neural models might successfully play the role of the oscillators in this model.

Previous oscillatory interference models that divided the oscillators among multiple cells (Burgess et al., 2007; Burgess, 2008; Hasselmo, 2008) assumed the postsynaptic cell $G$ integrated its inputs, despite $G$ often being identified with a resonant cell type: entorhinal cortex layer II stellate cells (Alonso and Klink, 1993; Giocomo et al., 2007). The distinction between resonators and integrators is a fundamental one that relates to the dynamics of the excitability of the neuron and affects the behavior of the cell in many ways (Izhikevich, 2007). Our present results suggest the oscillatory interference models might also work when the interference occurs in a resonant cell, supporting that previously untested assumption. It is interesting that the spiking of the resonate-and-fire cell in our simulations often occurred on alternate cycles (Fig. $7 C$, supplemental Fig. S5D; but see supplemental Fig. S7C, available at www.jneurosci.org as supplemental material), similarly to reports in ventrally located grid cells (Deshmukh et al., 2009).

We have made no explicit claims about the anatomical location or cell type of the oscillator networks or the grid cell proper. In principle, both parts of the model could be anywhere upstream of entorhinal cortex layer II (ECII), where the strongest grid signal to date has been found. In ECII, there is a dorsoventral gradient of grid field spacing and it is worth considering how this may relate to the model. In the present model, the intrinsic properties of the grid cell $G$ do not affect the spacing of the grid fields. Instead, the spacing depends on the velocity-to-frequency transformation performed by both the velocity inputs and the oscillator networks. One possibility is that there are separate velocity signals for each VCO spatial scale. Alternatively, a single output signal might go to all VCO networks and a difference in spacing would come from changes in the $F(I)$ slope (shallower ventral) over the range of velocity signal levels. One way to decrease the slope (increase spacing) consistent with the dorsoventral gradient along ECII (Hafting et al., 2005) is to increase the synaptic coupling in the network (Fig. $1 B$ ), which is consistent with increased temporal summation of EPSPs in ventral stellate cells (Garden et al., 2008). Another successful way to change the $F(I)$ slope is to proportionally scale down all the conductance densities in the model (e.g., increase $C_{\mathrm{m}}$ ), though other ways of changing the slope are known (Mehaffey et al., 2005; Fernandez and White, 2010).

Simplifications made in this work sidestepped many remaining issues in creating completely realistic oscillatory interference models. Cell-to-cell variations of membrane and synaptic conductances can be very high (Goaillard et al., 2009), and increasing oscillator heterogeneity decreases synchrony (Winfree, 1967; Cumin and Unsworth, 2007; Galán et al., 2007). Similarly, the inputs to individual VCO neurons themselves may be nonuniform, which can also interfere with synchronization (Golomb and Rinzel, 1993; Tsodyks et al., 1993; Wang and Buzsáki, 1996; White et al., 1998). Individual cells may receive inputs from dif- 
ferent numbers of velocity-coding cells with different firing rates and with different synaptic strengths, and the result of these nonuniformities on synchrony and the frequency response of the network is difficult to predict. In contrast, homeostatic mechanisms might cause each cell to adjust its membrane properties to produce the same $F(I)$ curve as other cells, despite dissimilar profiles of membrane conductances.

Another relevant issue is that correlated noise can synchronize oscillators (stochastic synchrony), particularly when their frequencies are similar (Teramae and Tanaka, 2004; Galán et al., 2007; Nakao et al., 2007; Marella and Ermentrout, 2008; Ly and Ermentrout, 2009). Noise in the body velocity signal might apply pressure toward synchronization of the VCO networks with each other and away from the correct phase differences (in addition to the spatial phase errors that will arise from the velocity noise itself). Further, correlated noise to neurons within networks $V_{\mathrm{i}}$ cannot be corrected through coupling, so even a small amount may have a noticeable effect on the stability of the system.

An as yet unanswered question about the grid network in animals is how many distinct spatial scales are represented (but see Barry et al., 2007). In single-cell oscillatory interference models, each grid cell can have a unique spatial scale. Continuous attractor models, however, require networks of grid cells that have identical spatial scales. With the present model, arguments of parsimony (using as few cells as possible) suggest that because large networks are needed to provide stable oscillations, there will likely be fewer VCOs than grid cells. Many grid cells would then share the same VCOs and so would be of the same spatial scale.

Our simulations provide a number of novel predictions that might be tested experimentally. First, we predict that an oscillator's input should come from cells with activity that is the inverse of the VCO's $F(I)$ curve, with a velocity relation like that shown in Figure $1 C$. Second, when an animal makes a sudden reversal of direction (e.g., on a linear track), the firing rate adaptation may be detectable as slight changes in grid position in proportion to the animal's speed and angle of motion compared with the grid cell orientation. Also, if VCOs are shared among grid cells, drifts in the spatial firing of individual cells may be partially correlated (in proportion to the number of VCOs shared and along the directions of the shared inputs). This is in contrast to a continuous attractor network where drift among all neighboring cells would be highly correlated (Burak and Fiete, 2009) or other oscillatory interference models where the drift of each cell is independent (Zilli et al.,2009). Finally, this model may be directly tested through the use of single-cell intracellular in vivo recording, which is increasingly feasible (Harvey et al., 2009; Lee et al., 2009). Our model predicts that synaptic potentials recorded in the cell generating the grid pattern should resemble the postsynaptic traces we have shown in Figure 7 and supplemental Figures S5 and S7. Specifically, each grid cell should always be receiving at least three strong rhythmic inputs that change their phase relative to each other according to the animal's spatial position. Of all the model predictions, this is the most important as it offers a direct, falsifiable test of this model.

We have provided a successful biophysical simulation of an oscillatory interference grid cell model and demonstrated its robustness against realistic levels of noise. This provides a proof of concept that suggests biological systems can implement the oscillatory interference model despite the high levels of intrinsic neuronal noise, but only through experimental tests designed to distinguish between models will the true mechanism underlying the grid pattern be discovered.

\section{References}

Acker CD, Kopell N, White JA (2003) Synchronization of strongly coupled excitatory neurons: relating network behavior to biophysics. J Comput Neurosci 15:71-90.

Alonso A, Klink R (1993) Differential electroresponsiveness of stellate and pyramidal-like cells of medial entorhinal cortex layer II. J Neurophysiol 70:128-143.

Ang CW, Carlson GC, Coulter DA (2005) Hippocampal CA1 circuitry dynamically gates direct cortical inputs preferentially at theta frequencies. J Neurosci 25:9567-9580.

Barry C, Hayman R, Burgess N, Jeffery KJ (2007) Experience-dependent rescaling of entorhinal grids. Nat Neurosci 10:682-684.

Blair HT, Welday AC, Zhang K (2007) Scale-invariant memory representations emerge from moiré interference between grid fields that produce theta oscillations: a computational model. J Neurosci 27:3211-3229.

Blair HT, Gupta K, Zhang K (2008) Conversion of a phase- to rate-coded position signal by a three-stage model of theta cells, grid cells, and place cells. Hippocampus 18:1239-1255.

Burak Y, Fiete IR (2009) Accurate path integration in continuous attractor network models of grid cells. PLoS Comput Biol 5:e1000291.

Burgess N (2008) Grid cells and theta as oscillatory interference: theory and predictions. Hippocampus 18:1157-1174.

Burgess N, Barry C, O’Keefe J (2007) An oscillatory interference model of grid cell firing. Hippocampus 17:801-812.

Chapman CA, Lacaille JC (1999) Intrinsic theta-frequency membrane potential oscillations in hippocampal CA1 interneurons of stratum lacunosum-moleculare. J Neurophysiol 81:1296-1307.

Couey JJ, Witter MP (2010) Local synaptic organization of the entorhinal cortex layer II network. FENS Abstr 5:105.4.

Cumin D, Unsworth C (2007) Generalising the Kuramoto model for the study of neuronal synchronisation in the brain. Physica D 226:181-196.

Deshmukh SS, Yoganarasimha D, Voicu H, Knierim JJ (2009) Thetaskipping in the medial entorhinal cortex. Soc Neurosci 35:100.5.

Dhillon A, Jones RS (2000) Laminar differences in recurrent excitatory transmission in rat entorhinal cortex in vitro. Neuroscience 99:413-422.

Dickson CT, Magistretti J, Shalinsky MH, Fransén E, Hasselmo ME, Alonso A (2000) Properties and role of $\mathrm{I}(\mathrm{h})$ in the pacing of subthreshold oscillations in entorhinal cortex layer II neurons. J Neurophysiol 83:2562-2579.

Erchova I, Kreck G, Heinemann U, Herz AV (2004) Dynamics of rat entorhinal cortex layer II and III cells: characteristics of membrane potential resonance at rest predict oscillation properties near threshold. J Physiol 560:89-110.

Fernandez FR, White JA (2008) Artificial synaptic conductances reduce subthreshold oscillations and periodic firing in stellate cells of the entorhinal cortex. J Neurosci 28:3790-3803.

Fernandez FR, White JA (2010) Gain control in CA1 pyramidal cells using changes in somatic conductance. J Neurosci 30:230-241.

Fransén E, Alonso AA, Dickson CT, Magistretti J, Hasselmo ME (2004) Ionic mechanisms in the generation of subthreshold oscillations and action potential clustering in entorhinal layer II stellate neurons. Hippocampus 14:368-384.

Fuhs MC, Touretzky DS (2006) A spin glass model of path integration in rat medial entorhinal cortex. J Neurosci 26:4266-4276.

Fyhn M, Molden S, Witter MP, Moser EI, Moser MB (2004) Spatial representation in the entorhinal cortex. Science 305:1258-1264.

Galán RF, Ermentrout GB, Urban NN (2007) Stochastic dynamics of uncoupled neural oscillators: Fokker-Planck studies with the finite element method. Phys Rev E Stat Nonlin Soft Matter Phys 76:056110.

Garden DL, Dodson PD, O’Donnell C, White MD, Nolan MF (2008) Tuning of synaptic integration in the medial entorhinal cortex to the organization of grid cell firing fields. Neuron 60:875-889.

Gaussier P, Banquet JP, Sargolini F, Giovannangeli C, Save E, Poucet B (2007) A model of grid cells involving extra hippocampal path integration, and the hippocampal loop. J Integr Neurosci 6:447-476.

Giocomo LM, Hasselmo ME (2008) Computation by oscillations: implications of experimental data for theoretical models of grid cells. Hippocampus 18:1186-1199.

Giocomo LM, Zilli EA, Fransén E, Hasselmo ME (2007) Temporal frequency of subthreshold oscillations scales with entorhinal grid cell field spacing. Science 315:1719-1722.

Glasgow SD, Chapman CA (2007) Local generation of theta-frequency EEG activity in the parasubiculum. J Neurophysiol 97:3868-3879. 
Goaillard JM, Taylor AL, Schultz DJ, Marder E (2009) Functional consequences of animal-to-animal variation in circuit parameters. Nat Neurosci 12:1420-1428.

Golomb D, Rinzel J (1993) Dynamics of globally coupled inhibitory neurons with heterogeneity. Phys Rev E Stat Phys Plasmas Fluids Relat Interdiscip Topics 48:4810-4814.

Guanella A, Kiper D, Verschure P (2007) A model of grid cells based on a twisted torus topology. Int J Neural Syst 17:231-240.

Haas JS, White JA (2002) Frequency selectivity of layer II stellate cells in the medial entorhinal cortex. J Neurophysiol 88:2422-2429.

Hafting T, Fyhn M, Molden S, Moser MB, Moser EI (2005) Microstructure of a spatial map in the entorhinal cortex. Nature 436:801-806.

Harvey CD, Collman F, Dombeck DA, Tank DW (2009) Intracellular dynamics of hippocampal place cells during virtual navigation. Nature 461:941-946.

Hasselmo ME (2008) Grid cell mechanisms and function: contributions of entorhinal persistent spiking and phase resetting. Hippocampus 18:12131229.

Hasselmo ME, Giocomo LM, Zilli EA (2007) Grid cell firing may arise from interference of theta frequency membrane potential oscillations in single neurons. Hippocampus 17:1252-1271.

$\mathrm{Hu}$ H, Vervaeke K, Storm JF (2002) Two forms of electrical resonance at theta frequencies, generated by $\mathrm{M}$-current, h-current and persistent $\mathrm{Na}+$ current in rat hippocampal pyramidal cells. J Physiol 545:783-805.

$\mathrm{Hu}$ H, Vervaeke K, Graham LJ, Storm JF (2009) Complementary theta resonance filtering by two spatially segregated mechanisms in CA1 hippocampal pyramidal neurons. J Neurosci 29:14472-14483.

Huhn Z, Orbán G, Erdi P, Lengyel M (2005) Theta oscillation-coupled dendritic spiking integrates inputs on a long time scale. Hippocampus 15:950-962.

Izhikevich EM (2001) Resonate-and-fire neurons. Neural Netw 14:883894.

Izhikevich EM (2003) Simple model of spiking neurons. IEEE Trans Neural Netw 14:1569-1572.

Izhikevich EM (2007) Dynamical systems in neuroscience: the geometry of excitability and bursting. Cambridge, MA: MIT.

Jarsky T, Roxin A, Kath WL, Spruston N (2005) Conditional dendritic spike propagation following distal synaptic activation of hippocampal CA1 pyramidal neurons. Nat Neurosci 8:1667-1676.

Jeewajee A, Barry C, O’Keefe J, Burgess N (2008) Grid cells and theta as oscillatory interference: electrophysiological data from freely moving rats. Hippocampus 18:1175-1185.

Kropff E, Treves A (2008) The emergence of grid cells: intelligent design or just adaptation. Hippocampus 18:1256-1269.

Lee AK, Epsztein J, Brecht M (2009) Head-anchored whole-cell recordings in freely moving rats. Nat Protocols 4:385-392.

Lengyel M, Szatmáry Z, Erdi P (2003) Dynamically detuned oscillations account for the coupled rate and temporal code of place cell firing. Hippocampus 13:700-714.

Leung LS, Yu HW (1998) Theta-frequency resonance in hippocampal CA1 neurons in vitro demonstrated by sinusoidal current injection. J Neurophysiol 79:1592-1596.

Ly C, Ermentrout GB (2009) Synchronization dynamics of two coupled neural oscillators receiving shared and unshared noisy stimuli. J Comput Neurosci 26:425-443.

Ly C, Ermentrout GB (2010) Coupling regularizes individual units in noisy populations. Phys Rev E Stat Nonlin Soft Matter Phys 81:011911.

Magistretti J, Alonso A (1999) Biophysical properties and slow voltagedependent inactivation of a sustained sodium current in entorhinal cortex layer-II principal neurons a whole-cell and single-channel study. J Gen Physiol 114:491-509.

Magistretti J, Alonso A (2002) Fine gating properties of channels responsible for persistent sodium current generation in entorhinal cortex neurons. J Gen Physiol 120:855-873.
Manor Y, Rinzel J, Segev I, Yarom Y (1997) Low-amplitude oscillations in the inferior olive: a model based on electrical coupling of neurons with heterogeneous channel densities. J Neurophysiol 77:2736-2752.

Marella S, Ermentrout GB (2008) Class-II neurons display a higher degree of stochastic synchronization than class-I neurons. Phys Rev E Stat Nonlin Soft Matter Phys 77:041918.

McNaughton BL, Battaglia FP, Jensen O, Moser EI, Moser MB (2006) Path integration and the neural basis of the 'cognitive map'. Nat Rev Neurosci 7:663-678.

Mehaffey WH, Doiron B, Maler L, Turner RW (2005) Deterministic multiplicative gain control with active dendrites. J Neurosci 25:9968-9977.

Nakao H, Arai K, Kawamura Y (2007) Noise-induced synchronization and clustering in ensembles of uncoupled limit-cycle oscillators. Phys Rev Lett 98:184101.

Needleman DJ, Tiesinga P, Sejnowksi TJ (2001) Collective enhancement of precision in networks of coupled oscillators. Physica D 155:324-336.

Nordlie E, Gewaltig MO, Plesser HE (2009) Towards reproducible descriptions of neuronal network models. PLoS Comput Biol 5:e1000456.

O'Keefe J, Burgess N (2005) Dual phase and rate coding in hippocampal place cells: theoretical significance and relationship to entorhinal grid cells. Hippocampus 15:853-866.

O'Keefe J, Recce ML (1993) Phase relationship between hippocampal place units and the EEG theta rhythm. Hippocampus 3:317-330.

Pape HC, Paré D, Driesang RB (1998) Two types of intrinsic oscillations in neurons of the lateral and basolateral nuclei of the amygdala. J Neurophysiol 79:205-216.

Prescott SA, Ratté S, De Koninck Y, Sejnowski TJ (2008) Pyramidal neurons switch from integrators in vitro to resonators under in vivo-like conditions. J Neurophysiol 100:3030-3042.

Redish AD (1999) Beyond the cognitive map: from place cells to episodic memory. Cambridge, MA: MIT.

Redish AD, Touretzky DS (1997) Cognitive maps beyond the hippocampus. Hippocampus 7:15-35.

Remme MW, Lengyel M, Gutkin BS (2009) The role of ongoing dendritic oscillations in single-neuron dynamics. PLoS Comput Biol 5:e1000493.

Samu D, Eros P, Ujfalussy B, Kiss T (2009) Robust path integration in the entorhinal grid cell system with hippocampal feed-back. Biol Cybern 101:19-34.

Sargolini F, Fyhn M, Hafting T, McNaughton BL, Witter MP, Moser MB, Moser EI (2006) Conjunctive representation of position, direction, and velocity in entorhinal cortex. Science 312:758-762.

Tabareau N, Slotine JJ, Pham QC (2010) How synchronization protects from noise. PLoS Comput Biol 6:e1000637.

Teramae JN, Tanaka D (2004) Robustness of the noise-induced phase synchronization in a general class of limit cycle oscillators. Phys Rev Lett 93:204103.

Tsodyks M, Mitkov II, Sompolinsky H (1993) Pattern of synchrony in inhomogeneous networks of oscillators with pulse interactions. Phys Rev Lett 71:1280-1283.

Wang XJ, Buzsáki G (1996) Gamma oscillation by synaptic inhibition in an interneuronal network model. J Neurosci 16:6402-6413.

Welinder PE, Burak Y, Fiete IR (2008) Grid cells: the position code, neural network models of activity, and the problem of learning. Hippocampus 18:1283-1300.

White JA, Chow CC, Ritt J, Soto-Treviño C, Kopell N (1998) Synchronization and oscillatory dynamics in heterogeneous, mutually inhibited neurons. J Comput Neurosci 5:5-16.

Winfree AT (1967) Biological rhythms and the behavior of populations of coupled oscillators. J Theor Biol 16:15-42.

Zilli EA, Yoshida M, Tahvildari B, Giocomo LM, Hasselmo ME (2009) Evaluation of the oscillatory interference model of grid cell firing through analysis and measured period variance of some biological oscillators. PLoS Comput Biol 5:e1000573. 